\title{
Die europäische Verschuldungskrise: eine dreifach unerledigte Agenda
}

von Joachim Jens Hesse

\section{Einleitung}

Die gegenwärtige Krisenpolitik im Rahmen der Europäischen Union richtet sich kurzfristig auf die Lösung der im Fall Griechenlands besonders illustrativen Verschuldungsprobleme, mittelfristig auf die angesichts destabilisierender finanzwirtschaftlicher Arrangements eingeschränkte Nachhaltigkeit von Wirtschaftsstrukturen und langfristig auf eine Systemfrage, die zur Gewährleistung von Stabilität, Funktionalität und Legitimität des europäischen Regierungssystems auf Konsequenzen für die Interaktion von nationalstaatlicher und europäischer Ebene drängt. Die nachfolgenden Ausführungen nehmen diese gleich dreifache Agenda auf und suchen sie, auch jenseits ihrer aktuellen Ausprägungen, problemlösend abzuarbeiten. Dabei geht es zum einen um erkennbar überforderte staatliche Akteure und sich damit verbindende suboptimale Politiken, zum anderen - am Beispiel des Bankensektors - um entscheidende Fragen an der Schnittstelle des öffentlichen und privaten Handelns, schließlich um die Weiterentwicklung der Europäischen Union. Dass die derzeit nationalstaatlich wie europäisch verfolgte „Politik des Ungefähren“ kaum ausreichen dürfte, der Problemsituation gerecht zu werden, deuten die aktuellen Auseinandersetzungen an, wobei der Status quo eher durch Zeitgewinn und Anpassungsstrategien denn durch eine strukturell greifende Problemlösung gekennzeichnet scheint. Letztere steht im Vordergrund der Ausführungen, samt der sich damit verbindenden Konsequenzen für die Akteure, wobei es entgegen dem ubiqitär angebotenen ,Mehr an Europa“ um ein „,besseres Europa“ geht. Die Lösung des aktuellen Griechenland-Problems sollte jenseits des situativen Zeitgewinns den Weg zu einer dauerhaft tragfähigen europäischen Währungsunion weisen. Dass dies auch systemkritische Fragen aufwirft, wird seit geraumer Zeit selbst jenen deutlich, die hinter entsprechenden Überlegungen bislang ,unangemessene Dramatisierungen“ oder gar „euroskeptisches Denken“ vermuten. 


\section{II. Überforderter Staat - überfordertes Europa}

Summiert man die laufende Diskussion, orientiert sich die aktuelle Krisenpolitik fast durchgehend an kurzfristigen Überlegungen, die im Wesentlichen den durch Informationsmängel begrenzten Handlungsstrategien der einzelnen meist nationalstaatlich basierten Akteure entspringen und im Rahmen der europäischen Verhandlungsprozesse einem erweiterten Bedarf an Kompromissfindung unterliegen. Die durch Koalitionskonstellationen und potentiellen Wählerdruck geprägte binnenstaatlich-demokratische Logik wird in diesem Kontext durch europapolitische Grundhaltungen ergänzt, in der sich Selbstrechtfertigungen mit dem Versuch zur eigenen Bedeutungssteigerung verbinden, was die Drucksituation für alle Beteiligten erhöht und arbeitsteilige Problemlösungen erschwert.

So droht dem überforderten Staat ${ }^{1}$ ein überfordertes Europa gegenüber zu stehen, verkürzen sich die erkennbaren Politiken auf eine „Vermeidung von Staatsinsolvenzen um jeden Preis“, auf die Sicherung des heimischen Bankensektors und auf die Aufrechterhaltung der Währungsunion samt der aus ihr zu ziehenden Vorteile. Dabei addieren sich frühere Fehler (vor allem im Rahmen des Stabilitäts- und Wachstumspaktes), eingeschränkte Reagibilität (angesichts der in Teilen neuen Problemausprägungen) und schließlich divergente Rationalitätsvorstellungen zu einer individuellen wie kollektiven Überforderung der staatlichen und europäischen Akteure. Analytischer formuliert treten zu Anreizproblemen (im Prozess der Politikformulierung) Verflechtungsprobleme (bei der politischen Entscheidung) und Koordinationsprobleme (bei der politischen Umsetzung), kommt es in der Folge zu systemgefährdenden Prozessen und diskussionswürdigen Ergebnissen/Wirkungen. ${ }^{2}$ Dies wird erkennbar dadurch verstärkt, dass der strukturell vorgegebene Politikmodus vor allem einer (zunehmend aussichtsloseren) Beruhigung der „Märkte“ bzw. des breiten Publikums dient und man über Ankündigungspolitiken und Absichtserklärungen die benannte Zeit zu gewinnen sucht, ohne sie effektiv zu nutzen.

1 So bereits Ellwein, T./Hesse, J.J.: Der überforderte Staat, Baden-Baden, 1994/Frankfurt a. M., 1997.

2 Im Zeitablauf nachgezeichnet u.a. bei Hesse, J.J./Wright, V. (Hg.): Federalising Europe? The Costs, Benefits and Preconditions of Federal Political Systems, Oxford, 1996; ders./Grimm, D./Jochimsen, R./Scharpf, F.W.: Zur Neuordnung der Europäischen Union. Die Regierungskonferenz 1996/97, BadenBaden, 1997; ders./Bruha, T./Nowak, C. (Hg.): Welche Verfassung für Europa?, Baden-Baden, 2001; ders.: Wider den Hochmut und die Gleichgültigkeit: die Professionalisierung Europas als Zukunftsaufgabe, in: ZSE 3/4 (2005), 620-649; ders.: Vom Werden Europas. Der EU-Verfassungskonvent: Verfahren, Ansatz, Ergebnisse, Berlin, 2007; ders./Dreier, H./Graf, F.W. (Hg.): Staatswissenschaften und Staatspraxis, Baden-Baden, 2011. 


\section{Der Fall Griechenland: materielle Grenzen, fehlende Nachhaltig- keit, situative Reflexe}

Dass die griechische Staatsverschuldung überhaupt zu einem Problem wurde, ist bekanntlich der (aus heutiger Sicht) unverantwortlichen Zulassung des Landes zur Euro-Gruppe sowie der Nichtanwendung der Regeln des erkennbar gescheiterten (und wohl auch ab ovo falsch gedachten) Stabilitätspakts geschuldet. Heute ergibt sich die inzwischen nicht mehr bestreitbare Ausgangssituation, nach der das Land mit Schulden in Höhe von ca. 350 Mrd. Euro belastet ist, von denen angesichts der verfügbaren Rahmendaten niemand annehmen kann, dass sie jemals aus eigener Kraft abgetragen werden.

Im Gegenteil: Die ausgesandte Troika aus Europäischer Kommission, Europäischer Zentralbank (EZB) und Internationalem Währungsfonds (IWF) zeigte sich lange Zeit zögerlich, zur Vermeidung einer Insolvenz weitere $8 \mathrm{Mrd}$. Euro aus dem Hilfspaket der EU-Staaten und des IWF freizugeben. Geplant war, mit Hilfe eines drastischen Sparkurses das Haushaltsdefizit auf 7,6 Prozent des BIP zu drücken, eine auch angesichts der rasanten Schrumpfung der griechischen Wirtschaft offenkundig unhaltbare Vorgabe. Jetzt wird spekuliert, dass das Defizit bei über neun Prozent liegt, die Verschuldung des Landes mithin weiter wächst: von prognostizierten 166 Prozent des BIP in diesem auf vermutlich 173 Prozent im kommenden Jahr. Entsprechend wächst der Hilfebedarf, den die Troika um zusätzliche $100 \mathrm{Mrd}$. Euro korrigieren musste.

Zudem scheiterten zahlreiche der angekündigten Privatisierungspolitiken, blieb die Überprüfung des öffentlichen Dienstes bis hin zur Schließung fragwürdiger Staatsbetriebe und zur Rückführung von Personal ohne erkennbare Ergebnisse und gelingt es aufgrund einer hochdefizitären Finanzverwaltung und in Teilen inexistenter Katasterämter kaum, erweiterte Besteuerungsmaßnahmen umzusetzen oder gar ausstehende Steuerschulden einzutreiben. Letzteres wird in Berichten deutlich, nach denen die Bürger Griechenlands aufgrund rechtskräftiger Steuerbescheide ihrem Staat nicht weniger als 41 Mrd. Euro schulden, wobei durchaus auch staatliche Einrichtungen (wie etwa die Eisenbahn und weitere Verkehrsbetriebe) zu den großen Schuldnern zählen. Addiert man dem die starren Regeln für Berufsgruppen und die seit Jahren bekannte Tatsache, dass im griechischen Parlament verabschiedete Gesetze häufig vollzugslos bleiben, wird deutlich, dass es sich in diesem gleichsam exemplarischen Fall um im Wortsinne vormoderne Interaktions- und Verhaltensformen innerhalb wie zwischen Staat, Privatwirtschaft und der Bevölkerung handelt. 
Die seitens der Europäischen Union und des IWF oktroyierten Sparbemühungen suchen daher auch eher die nicht-griechische Politik und das breitere Publikum zu beruhigen, als dass sie problemlösende Wirkungen entfalten. In der Folge münden die inzwischen wohl dauerhaft rezessiven bzw. depressiven Tendenzen im griechischen Wirtschaftssystem in soziale Verwerfungen mit weiter destabilisierender Konsequenz. So werden den Namen verdienende Wachstumsprozesse unmöglich gemacht, gehen die am deutschen Modell einer „Schrumpfkur“ angelehnten Bemühungen um eine angebotsseitige reale Abwertung und eine Senkung von Lohnkosten fehl, da zum einen die Probleme wesentlich tiefgreifender und umfassender sind als im deutschen Fall und es zum zweiten an konfliktmoderierenden Einrichtungen, auch an handlungsfähigen großen Gewerkschaftsverbänden, mangelt. Im Ergebnis gelingt es gerade nicht, sachkundige Marktteilnehmer zu beruhigen, die in der Regel langfristig denken und den unzureichenden Ansatz wie die fehlende Konsequenz des Vorgehens erkennen was wiederum in einen circulus vitiosus münden könnte, der über ein weiteres Absinken der ökonomischen Basisindikatoren, eine Zementierung angebotener Hilfsmaßnahmen (wie des „Rettungsschirms“) und steigenden sozialen Widerstand eine Überforderung der Beteiligten bewirkt, die über Griechenland hinauszugehen droht. Irland und Portugal, ggf. auch Italien, Spanien und vielleicht sogar Belgien dürften in absehbarer Zeit weitere Kredithilfen benötigen. Von einer nachhaltigen Problemlösung kann angesichts solch kurzfristig ansetzender, unter den benannten Voraussetzungen eben nur begrenzt „rationaler“ Strategien sicher nicht gesprochen werden.

\section{Ein selbstreferentielles Bankensystem?}

Bezieht sich dieses Urteil auf die Verschuldungskrise, mithin die Wirkungsweise der öffentlichen Hand, ist Ähnliches für den Bankensektor zu diagnostizieren. Entsprechend divergent sind die Vorstellungen, wie künftig vorzugehen ist, mit Ausnahme des ersten „Ausfalls“, der belgisch-französischen Großbank Dexia, die in beträchtlichem Ausmaß Kredite an Griechenland vergab und jetzt verstaatlicht werden soll. Darüber hinaus kamen die deutsche Bundeskanzlerin und der französische Staatspräsident überein, ,das Nötige zu tun“, um eine Rekapitalisierung der Banken sicherzustellen. Im Hintergrund steht die Befürchtung, dass die Kapitaldecke zahlreicher großer Institute im Fall einer Insolvenz Griechenlands nicht ausreichen würde, die Verluste aufzufangen. Gerade deshalb gewinnen Vorstellungen von einem ,,Schuldenschnitt“ an Bedeutung, wobei die benannten Größenordnungen von einem Schnitt in Höhe von 50 bis 80 Prozent ausgehen, 
eine vor allem für die französischen Banken erschreckende Perspektive. Deutscherseits wird betont, dass der Bankensektor diesen Verlust im Falle Griechenlands zwar überstehen könnte, eine bei einer Ausweitung der Krise auf Spanien oder gar Italien allerdings nicht zu verallgemeinernde Aussage. Im Gegensatz zu Griechenland hat man in diesen Fällen einen etwaigen Schuldenschnitt noch nicht verbucht, auch geht es hier um ganz andere Größenordnungen. Als ein weiteres Risiko werden die sog. Kreditausfallversicherungen bezeichnet, die im Fall einer Staatsinsolvenz ausgezahlt werden. Man kann sie nicht an der Börse kaufen, Banken und Hedgefonds handeln sie untereinander. Die Preise für diese Versicherungen auf griechische Staatsanleihen sind in den letzten Jahren beträchtlich angestiegen - und niemand weiß, wer im Falle eines Schuldenschnitts dafür aufkommen wird. Als noch problematischer gilt ein solches Szenario für die spanischen Sparkassen, von denen einige über nur wenig Eigenkapital verfügen.

Aus der erkennbaren Verflechtung von Verschuldungskrise und Bankenrettung verdichten sich die Anzeichen, vor allem ,systemrelevante“ Institute zum Vorhalten einer verstärkten Eigenkapitaldecke zu veranlassen. In einem Versuch, den Primat der Politik gegenüber selbstreferentiellen Finanzmärkten zurückzugewinnen, geht es darum, die Staaten ,aus der Geiselhaft der Banken“ zu befreien. Im Fokus stehen hier Großbanken wie Goldman Sachs, JPMorganChase, HSBC, BNP Paribas und durchaus auch die Deutsche Bank, die nach einem Vorschlag des Financial Stability Board (FSB) ab dem Jahr 2016 ein zusätzliches Kernkapital i.H.v. 1-2,5 Prozent vorhalten sollten. Ergänzt man dies um die Basel III-Vorkehrungen (eine Kernkapitalquote von 7 Prozent bis zum Jahr 2019), ergeben sich jene 9 Prozent, die seit kurzem auch der EUKommissionspräsident von den Banken fordert. Allerdings handelt es sich erneut nur um mögliche Optionen; konkrete Beschlüsse werden erst anlässlich eines erneuten EU-Gipfels und schließlich in Cannes (G-20-Treffen) Anfang November dieses Jahres erwartet. Hier rechnet man dann auch mit ,ernsthafteren“ Maßnahmen, die sich jetzt nicht nur an die Großbanken, sondern auch an Versicherungen und andere Akteure als Adressaten der geplanten Reformmaßnahmen richten. Nach Auffassung der Bundesregierung müsste das auch die sog. Schattenbanken einbeziehen, um ein Ausweichen von Investoren auf weniger regulierte Akteure unmöglich zu machen. ${ }^{3}$

3 Daten aus der aktuellen Berichterstattung von Einrichtungen der Europäischen Union, der Bundesregierung sowie ausgewählter europäischer und deutscher Tageszeitungen. 
Gleichzeitig wird erkennbar versucht, weniger neue Einrichtungen schaffen zu wollen, als vielmehr durch Aufstockung der Mittel für bestehende Institutionen der Krise Herr zu werden. Dies geht bis hin zu der nun doch erwogenen Mittelerhöhung für den IWF, dessen Potential zur Überwachung der nationalen Wirtschaftspolitiken zudem gestärkt werden soll. Auch können sich einzelne Akteure eine erweiterte Zusammenarbeit zwischen dem IWF und regionalen Einrichtungen, wie dem EFSF, durchaus vorstellen, ergänzt um die Ermöglichung weiterer kurzfristiger Liquiditätshilfen für Krisenländer. In diesem Kontext ist bemerkenswert, dass und in welchem Ausmaß Schwellenländer weiter an Bedeutung gewinnen. Dies gilt natürlich vor allem für China, Brasilien und Indien, die im Gegenzug zu einer Erhöhung von IWF-Mitteln ihre Mitspracherechte auszuweiten suchen, eine für die USA wie die großen europäischen Länder noch nicht akzeptable Position.

Bei all dem ist schließlich zu berücksichtigen, dass der Widerstand des Bankensektors beträchtlich ist und an Bedeutung noch zunehmen dürfte. Dies kann auch als Antizipation einer weiteren Verschärfung der Diskussion gesehen werden, wie sie etwa seitens der politischen Opposition in Deutschland vorgetragen wird. Dies gilt vor allem für die geforderte Aufspaltung des Bankensektors in Geschäfts- und Investmentbanken sowie für das faktische Ausmaß der Gläubigerbeteiligung. Diese von populistischen Untertönen nicht freie Diskussion setzt im Kern daran an, die Staatshaftung zu begrenzen und künftig zu vermeiden, dass der Bankensektor seine Krisen auf die Allgemeinheit abzuwälzen vermag. Da entsprechende Politiken, seit dem Lehman-Zusammenbruch versprochen, bis heute nicht greifen, wird die Überhitzung der Debatte verständlich. Bedenkt man darüber hinaus, dass sich die durchgeführten Banken-Stresstests als weitgehend „Zahnlos" erwiesen haben, der Eigen- und Derivatehandel keiner nachhaltigen Regulierung zugeführt wurde und selbst die Einführung einer Transaktionssteuer bislang an innereuropäischen und transatlantischen Differenzen scheiterte, lässt sich das Ausmaß des anstehenden politischen Entscheidungsbedarfs erkennen.

Im Übrigen wären bei einem konsequenten Durchdenken der möglichen Optionen ,geordneten Staatsinsolvenzen“ durchaus auch Bankeninsolvenzen gegenüberzustellen, eine in der Logik höchst plausible, im Alltagshandeln allerdings noch beträchtlich umstrittene Erkenntnis. Schon mehren sich die Stimmen, die zwischen Milliardenhilfen für private Banken und deren endgültiger Verstaatlichung keinen großen Unterschied mehr sehen.

Angesichts dieser Ausgangssituation sprach sich das künftige EZB-Direktoriumsmitglied Asmussen zwischenzeitlich für eine gleichzeitige Rekapitalisie- 
rung aller wichtigen europäischen Banken aus, ein institutsspezifisches Vorgehen sei nicht sinnvoll. Zudem müsse eine Lösung für die Banken Bestandteil eines Gesamtpakets sein, da isolierte Instrumente in der gegenwärtigen Situation wenig hilfreich wären. Dieser Ansatz erscheint insofern vertretbar, als er die Angst vor Wettbewerbsnachteilen im Bankensektor zu akkomodieren sucht. Als ein Beispiel wird in diesem Kontext häufig auf die USA verwiesen, wo bekanntlich alle Großbanken eine staatliche Kapitalzufuhr annehmen mussten - mit dem Ergebnis, dass der Staat nach der Krise in zahlreichen Fällen sogar mit Gewinn wieder ,aussteigen“ konnte. Angesichts der bereits angesprochenen Skepsis des deutschen Bankensektors, nach der eine staatlich aufgezwungene Rekapitalisierung ,einer Verstaatlichung gleichkäme“, sucht die deutsche Regierung im Gegensatz zur französischen den Banken zunächst die Möglichkeit einzuräumen, sich die Gelder selbst am Kapitalmarkt zu beschaffen, bevor der Staat einspringt. Volumenmäßig geht man mit Blick auf diese Zwangskapitalisierung von Größenordnungen bis zu $50 \mathrm{Mrd}$. Euro aus, eine Summe, die beträchtlich über jenen 29 Mrd. liegt, die der Bund in der ersten Phase der Finanzmarktkrise zuschießen musste. Dies erklärt sich auch aus Überlegungen, ggf. alle Institute, mithin nicht nur „systemisch wichtige“ Großbanken (eine nie präzise definierte Kategorie), sondern auch Sparkassen und Volksbanken einer anspruchsvollen Eigenkapitalquote zu unterlegen.

Aus diesem Bündel unterschiedlicher, sich in Teilen widersprechender Einzelmaßnahmen wird deutlich, dass es auch mit Blick auf den Bankensektor um eher kurzfristig angelegte „Strategien“ der politisch Handelnden geht, von denen abzusehen ist, dass sie in ihren Konsequenzen noch nicht ausreichend durchdacht sind. Erneut wird damit das bereits im Fall Griechenlands angesprochene Politikmuster erkennbar: Es dominieren situative Reaktionen auf erkennbare Verwerfungen, wobei binnenstaatliche, europäische und bankenspezifische Reaktionsmuster in Teilen isoliert voneinander konzipiert werden. Dies begünstigt die Gefahr suboptimaler Politiken, verstärkt die Tendenz zu einem (hier systemisch relevanten) Eingriffsverzicht und tangiert schließlich eher den Steuerzahler als den Gläubiger.

\section{Das geringere Übel statt Scylla und Charybdis?}

Lassen sich Marktlogik, demokratische Binnenlogik und Europäische Gemeinschaftslogik trotz der benannten Bemühungen auf keinen gemeinsamen Nenner bringen, sind jenseits der aufgezeigten, situativ geprägten Politiken strukturelle Problemlösungen gefordert, da sich sonst sehr schnell Systemfragen stellen 
könnten. Diese reichen von einer berechtigten Infragestellung einzelner Aspekte des Wirtschaftssystems, etwa der bereits diskutierten Funktionslogik des Finanzsektors, über Generalkritiken am Modus einer ausschließlich wachstumsorientierten Marktwirtschaft (nicht zuletzt mit Blick auf die seit den 1990er Jahren entkoppelte Entwicklung von Löhnen und Kapitalrenditen) bis hin zu einer Infragestellung der Reagibilität und Legitimität der Europäischen Union sowie, im schlimmsten Fall, der sie konstituierenden repräsentativdemokratischen Systeme. Dass entsprechende Fragen sich zunehmend verdichten, dokumentieren die zwar ratlosen, aber verständlicherweise „empörten“ Proteste nicht nur in Griechenland, sondern auch in Spanien, Italien, Frankreich, Israel und zuletzt in New York, London und Frankfurt. Die benannte „Politik des Ungefähren“ löscht Feuer mit Öl, statt auf berechtigte Systemzweifel einzugehen und dem Souverän die daraus entstehenden Handlungsoptionen unmissverständlich und unter Berücksichtigung der absehbaren Folgewirkungen zu erklären. Dass etwa ein umfassender Schuldenschnitt im Fall Griechenlands zunehmend unvermeidbar wird, ist klar zu benennen. Dem schließt sich die Notwendigkeit einer offenen Diskussion proaktiver statt reaktiver Politikmuster an.

In der aktuellen Debatte wird der Anschein erweckt, als müsse man die Europäische Union um jeden Preis auf einen vertretbaren Mittelweg zwischen zwei politisch und ökonomisch katastrophalen outcomes lenken: der Scylla eines Eintritts in eine dauerhafte Fiskalunion einerseits und der Charybdis eines auch nur teilweisen Scheiterns der Eurozone andererseits. Vieles spricht jedoch dafür, dass die aktuelle Situation nur eine Wahl des kleineren Übels zulassen wird, weshalb eine nüchterne Diskussion der Handlungsoptionen umso angezeigter erscheint. Idealtypisch geht es im Wesentlichen um zwei gegensätzliche Vorgehensweisen, die je für sich mit hohen sozialen, ökonomischen, politischen und ggf. auch „strategischen“ Kosten verbunden sind. Sie seien im Folgenden skizziert und gegenübergestellt, ein Ansatz, der sich mit Blick auf demokratiepolitische Minima auch für die öffentliche Diskussion empfehlen dürfte.

\section{Die Fiskalunion als Einstieg in die Politische Union}

Die erste strukturell greifende Lösung kann als langfristige Akzeptanz der Fiskalunion bezeichnet werden und stellt im Ergebnis einen Einstieg in die die Wirtschafts- und Währungsunion ergänzende Politische Union dar. In der Konsequenz hießen die entscheidenden Elemente: Europäischer Finanzausgleich, Konzipierung und Gewährleistung von Eurobonds sowie gemeinsame Haftung der Mitgliedstaaten. Zwar ist erkennbar, dass sich vor allem die deutsche Seite 
gegen eine solche Fiskalunion noch wehrt, doch wächst der Druck, in diese Richtung zu denken und zu handeln; jüngste Äußerungen des Bundesfinanzministers gehen bereits in diese Richtung. ${ }^{4}$ Allerdings sollte man sich dabei auch der Konsequenzen bewusst sein, die die „Völker Europas“ als restringierender Faktor auszulösen vermögen.

In Verfolgung dieser Strategie würde den handelnden Akteuren in und für Griechenland ein Erhalt des Status quo ermöglicht, dabei allerdings vor allem der Norden Europas belastet und daher von beträchtlichen politischen Akzeptanzproblemen begleitet sein. So sollte sich herum gesprochen haben, dass die europäische Öffentlichkeit seit langem von einem Europa "North of Paris“ und „South of Paris “ spricht. Unterschiedliche Rationalitätsvorstellungen, Wertesysteme und Verhaltensnormen sind hier die Basis wechselseitiger Vorbehalte (und Vorurteile). Hinzu kommt, dass eine dauerhafte Alimentierung des Südens durch den Norden des Kontinents mittelfristig kaum umsetzbar sein dürfte. Damit sind nicht nur die Erfahrungen bei der Zurückweisung des Verfassungsvertrages angesprochen, sondern auch die entsprechenden Entscheidungen im Rahmen der Ratifizierung des Vertrags von Lissabon. Die dem zugrunde liegenden Beschlüsse und Sicherungen haben zwischenzeitlich zu nicht eben leicht zu überspringenden „Hürden“ geführt, zumal sich die vertrags- und verfassungsrechtliche Ausgangssituation durch die neue Rechtsprechung des Bundesverfassungsgerichts und politische Absichtserklärungen in anderen Mitgliedstaaten der Europäischen Union entscheidend verändert hat.

Zur damit verbundenen Erschwerung europabezogener Willensbildungs- und Entscheidungsprozesse träte funktional eine erneute Veränderung der Verträge, ein bekanntlich riskanter Weg, da das europäische Publikum der Veränderungsintensität müde geworden scheint und den Brüsseler Einrichtungen zu misstrauen beginnt. Kontinuierliche Neuerungen und mit „Sachzwang“ begründete weitere Souveränitätseinbußen werden inzwischen nicht mehr als flexible Anpassung an sich verändernde Rahmenbedingungen gesehen, sondern schlagen sich (auch und gerade an der Wahlurne) in Abwehrhaltungen, Renationalisierungsansätzen und der Rückbesinnung auf überschaubare Territorialitäten nieder. Der ubiquitäre Verweis auf eine potentiell „heilende“ Subsidiarität gilt zu Recht als pazifierend gedachte Kopfgeburt von Zentralisten. 
Hinzu treten Prozesse, die Analysten föderalstaatlicher Systeme, und hier vor allem der deutschen bundesstaatlichen Ordnung, seit Jahren beklagen und in ihrer Wirkung als leistungsmindernd einschätzen: der fehlende Anreiz, die eigene Wettbewerbsfähigkeit zu stärken, die wachsende Gewöhnung an Subsidien und eine Alimentierung durch Andere, die Exekutivlastigkeit des politischen Prozesses. Auch die gewiss polemische, gleichwohl aber realanalytisch treffende Metapher von einer „Dauersubventionierung eines europäischen Mezzogiorno“5 findet hier ihren Ursprung und ihre Berechtigung. Schließlich tritt meist eine beträchtliche Unkenntnis der sich mit einer solchen Systemtransformation verbindenden Kosten hinzu, wobei es eben nicht nur Transaktionskosten (im weiteren Sinne, also unter Einschluss der Aufwendungen für institutionelle Veränderungen) sind, die es zu berücksichtigen gilt, sondern vor allem jene erwartbaren Dauertransfers, die die ökonomische Leistungsfähigkeit wichtiger Akteure massiv beeinträchtigen könnten. Der vermeintliche „deutsche Egoismus“ bezeichnet in der Realität ein durchaus anerkennenswertes Bemühen, die für alle wirksam werdenden Stärken von Gemeinschaftsteilen oder einzelnen Akteuren nicht ohne Not zu schwächen, unabweisbare Leistungs- und erhoffte Verteilungsorientierungen mithin in ihrer Eigenständigkeit wie Komplementarität zu erkennen.

Im Ergebnis stünde entweder eine zumindest mittelfristig zum Scheitern verurteilte Vertragsrevision (sowie in einigen Mitgliedstaaten die rechtlich vorgeschriebene Verfassungsneuschöpfung) zur Begründung eines europäischen Bundesstaates oder aber die spill-over-getriebene schleichende Entwicklung hin zu einer de-facto-Transferunion, die die ohnehin kritisierte Glaubwürdigkeit repräsentativdemokratischer Systeme in ihren Grundfesten erschüttern könnte.

\section{Bestandssicherung und Wiederaufbau}

Der zweite, gleichsam alternative Ansatz sucht diesen folgenschweren Übergang in die Fiskalunion durch eine einmalige Reform der Euro-Gruppe und eine damit verbundene längerfristige Stabilisierung der Einzelstaaten zu vermeiden. Im Ergebnis verbänden sich hier Marktlogik mit demokratischer Binnen- und Europäischer Gemeinschaftslogik - in dieser Reihenfolge. Einzelne Elemente wären wiederum ein umfassender Schuldenerlass im griechischen Fall, der jetzt den Vorteil hätte, als Einmalbelastung eine dauerhafte Alimentierung des griechischen Staates und seiner Wirtschaft zu vermeiden. Dieser Schnitt wäre zu ergän-

5 Scharpf, F. W., in diesem Heft. 
zen durch den Austritt Griechenlands aus der Euro-Gruppe (nicht der EU), da nur eine Abwertung der griechischen Währung den notwendigen Wachstumsimpuls zu setzen vermag, eine endogene Quasi-Abwertung nach dem deutschen Modell hingegen kaum machbar erscheint. Griechenland würde also im Rahmen einer ,geordneten Insolvenz“ selbst aus der Euro-Gruppe austreten, die Landeswährung zurückgewinnen und über eine sofortige Abwertung seine Wettbewerbschancen auf den Weltmärkten zu verbessern suchen.

Den erkennbaren Vorteilen, auf diese Weise einer die eigenen Wirtschaftspotentiale gefährdenden Haftungsgemeinschaft zu entgehen und die Euro-Gruppe wie die EU als Ganzes zu stabilisieren, stünde allerdings eine Reihe möglicher Nachteile entgegen: ${ }^{6}$ Sie gelten zunächst für vertragsrechtliche Hürden und verbänden sich ferner mit dem, was unpräzise immer wieder als „Ansteckungsgefahr" oder "Dominoeffekt" in die Diskussion eingebracht wird: die etwaige Übertragung der griechischen Probleme auf Portugal, Irland oder andere EUMitgliedstaaten. Hier wäre zunächst darauf zu verweisen, dass die sehr unterschiedliche Ausgangssituation der EU-Mitgliedstaaten verallgemeinerungsfähige Aussagen erschweren sollte. Weder Portugal noch Irland und erst recht nicht Spanien und Italien sind durch eine mit Griechenland vergleichbare ökonomische, soziale oder auch politische Ausgangssituation gekennzeichnet. Stellt man etwa die Reaktionsmuster dieser Staaten in den vergangenen Monaten einander gegenüber, so werden die differenten institutionell-organisatorischen, prozessualen und materiell-inhaltlichen Ausprägungen der Wirtschafts- und Fiskalpolitik deutlich. Dies schließt natürlich nicht aus, dass Spekulanten versuchen werden, auch hier auf eine Verschärfung von Übergangsproblemen zu setzen, doch würde die Ergänzung einer entschiedenen Haltung im Fall Griechenlands durch kluge Stabilisierungsmaßnahmen in den übrigen Staaten der europäischen Peripherie dem entgegenwirken können, im Zweifel auch über die temporäre Stützung durch gemeinschaftlich gedeckte Anleihen. Im Gegensatz zu Griechenland verfügen die übrigen GIPS-Staaten über das Potential, ihre wirtschaftliche und fiskalische Lage innerhalb der Währungsunion zu stabilisieren.

Als problematischer für die Gesamtentwicklung der Europäischen Union könnte sich erweisen, dass im Fall Griechenlands Kapitalflucht, bank run und Bankeninsolvenzen in Prozesse münden, die bis hin zu einer Landflucht oder gar Massenmigration reichen könnten, mithin ein Infragestellen des Binnenmarktes (samt

6 Vgl. unter diversen diesbezüglichen Veröffentlichungen etwa UBS Investment Research: Euro Breakup: The Consequences, London, 2011. 
der ihm inhärenten Grundfreiheiten) bedeuteten. Der Autor sieht diesbezügliche Gefahren in diesem Ausmaß nicht, vor allem dann nicht, wenn sich die Mitgliedstaaten der Union auf ein „Wiederaufbauprogramm Griechenland“ verständigen, das sich auf eine Modernisierung der Infrastruktur des Landes (im weitesten Sinne) richtet.

Schließlich wird befürchtet, dass die unmittelbaren ökonomischen Folgen eines Austritts zu einer Destabilisierung des demokratischen Systems, einer Stärkung populistischer Parteien und schließlich zu sozialen Unruhen bis hin zur Gewaltanwendung führen könnte. Das sind gewiss Gefahren, die es zu berücksichtigen gilt, doch hat die Entfremdung im politischen System Griechenlands bereits heute ein Ausmaß angenommen, nach dem eine weitere Verschärfung der Ausgangssituation weniger wahrscheinlich ist. Im Gegenteil könnte die griechische Regierung durch einen gemeinsam zu schulternden nationalen Kraftakt ,gegen die Märkte" samt greifbarer Zukunftsperspektive und externer Hilfe die Unterstützung des demos zurückgewinnen, während das derzeitige Vorgehen den politisch Handelnden fortwährend abverlangt, dem Souverän extern oktroyierte Zumutungen schmackhaft zu machen. Hinzu kommt, dass die Europäische Union aus Eigeninteresse an einem verbleibenden Mitgliedstaat zu Stabilisierungsleistungen aufgefordert wäre. Das, was man etwa mit Blick auf vermeintlich problematische EU-Neumitglieder aus Mittel- und Osteuropa in den vergangenen beiden Jahrzehnten an Stabilisierungsleistungen erbrachte, ist nicht nur aller Ehren wert, sondern in Teilen auch auf weniger entwickelte oder sich in einer Transformationsphase befindliche „Altmitglieder“ zu übertragen. Potentieller Verfall der demokratischen Substanz und gewaltsame Auseinandersetzungen galten auch hier als beliebtes Argument, strukturell wirkungsmächtigen Problemlösungen zu begegnen, sie gleichsam abzuwehren, doch sind die späteren Erfolge „simulierten Polarisierungen“ im Rahmen der europapolitischen Diskussion entgegenzuhalten.

Auch die damit skizzierte Lösung wäre für alle Beteiligten kostenintensiv und nachteilsbehaftet. Demgegenüber könnte die dauerhafte Einrichtung von Ressourcentransfers zwar kurzfristig günstiger erscheinen, doch bliebe die Frage nach den langfristigen Entwicklungsperspektiven unbeantwortet. Der Austritt aus der Währungsunion gestattete den skizzierten „Neuanfang“ und wäre nach Erreichen vereinbarter Mindeststandards (und der Umsetzung notwendiger Reformen auch innerhalb der Eurozone) reversibel, während die Einrichtung eines permanenten Finanzausgleichs bestehende Unterschiede beim Entwicklungspotential und der Wettbewerbsfähigkeit dauerhaft zementieren dürfte. 


\section{Fazit}

Keiner der aufgezeigten Wege zur Lösung der Verschuldungskrise ist risikolos. Es wird deshalb darum gehen, jenen Weg zu identifizieren, der unnötige Kosten, vor allem aber eine Daueralimentierung des europäischen Südens vermeidet und den größten Grad an Realisierungswahrscheinlichkeit aufweist. Dabei gilt es wie stets, zwischen normativen und funktionalen Argumenten zu unterscheiden.

Während normativ das gewonnene Europa vielen als unauflösbar erscheint, man sich mithin jeder „Ausgrenzung“ verwehrt, sei daran erinnert, dass die Union bereits heute durch zahlreiche Gruppenbildungen geprägt ist, die sich spezifischen Interessen verdanken. Nicht nur die Euro- oder die Schengen-Gruppe verweisen darauf, dass es sich im Rahmen unterschiedlicher Integrationsstufen als durchaus wirksam erwiesen hat, Interessen oder auch Stärken und Schwächen ggf. zu bündeln, sie gleichwohl aber im Kontext eines gemeinsamen Rahmens wirksam werden zu lassen. Dies stellt sich in besonderem Maß für eine inzwischen 27 und in absehbarer Zukunft wohl 32 Mitglieder umfassende Union, der es ohnehin an Kohäsion und Kohärenz mangelt; sie in ihrer Gesamtheit dadurch zu gefährden, dass ein Mitgliedstaat sich aufgrund unzutreffender Informationen und missbräuchlicher Vorgehensweisen Vorteile verschafft, erscheint unverantwortbar. Hinzu treten jene strukturellen Defizite der Währungsunion, die diese Entwicklung begünstigt und in Teilen durchaus mit verursacht haben. Sie gilt es sorgfältig zu analysieren und aufzulösen.

Der EU dürfte vor allem dadurch gedient sein, dass man arbeitsfähige Gruppen akzeptiert und toleriert, die innerhalb eines Gesamtrahmens tätig werden und deren Mitglieder bei Fehlverhalten mit harten, also verpflichtenden Sanktionen zu rechnen haben. Umgekehrt ist es nicht nur aus der Logik des Arguments heraus wünschenswert, dass diese Gruppen für diejenigen Mitgliedstaaten offen bleiben, die aufgrund ihrer je eigenen Entwicklung und Interessenlage eine neue oder erweiterte Beteiligung wünschen. Die Europäische Union würde so zu einem Muster einer Regionalorganisation, die $\mathrm{Zu}$ - und Abgänge erlaubte, ohne jeweils das Gesamtgefüge zu gefährden.

In funktionaler Sicht verbände das hier skizzierte Vorgehen Leistungsorientierung mit Sanktionspotential, würde Verteilungspolitiken nicht ausschließen, sie aber zugunsten der Gesamtentwicklung zurückstehen lassen und erlaubte den „Völkern Europas“ eine Wiedergewinnung des zwischenzeitlich verlorenen Glaubens an die Arbeitsfähigkeit der europäischen Einrichtungen. Erneut: „Euroskepsis“ bildet sich nur dann, wenn die Ergebnisse gemeinschaftlichen Han- 
delns suboptimal bleiben, von unzureichend legitimierten technokratischen Eliten vertreten werden und letztlich in erweiterte Verteilungskämpfe münden. Niemand, der solches kritisiert, steht außerhalb des europäischen Gedankens. Dies anzuerkennen wäre aus Sicht allzu ,gläubiger“ Europäer sicher sinnvoll. Pragmatisch-konstruktive Lösungen, die trotz gelegentlicher Rückschritte für Einzelne das „europäische Haus“ als Ganzes stabilisieren, sollten daher falschen Kompromissen vorgezogen werden, die lediglich Umverteilungen bewirken und damit zusätzliche integrationsfeindliche Aversionen schüren. Der „Fall Griechenland" könnte in diesem Sinne eine durchaus reinigende Wirkung entfalten. 